\title{
Diurnal variations in glycaemic response to a low and high glycaemic index meal in elderly Chinese Singaporeans
}

\author{
L. Egli ${ }^{1}$, S. Haldar ${ }^{2}$, C.A. De Castro $^{3}$, C. Darimont ${ }^{1}$, K. Macé $^{1}$ and C.J.K. Henry ${ }^{2}$ \\ ${ }^{1}$ Nestle Research Centre, Lausanne, Switzerland, \\ ${ }^{2}$ Clinical Nutrition Research Centre, Singapore Institute for Clinical Sciences, Agency for Science Technology and \\ Research $\left(A^{*} S T A R\right)$, Singapore and \\ ${ }^{3}$ Nestle Research Centre Asia, Singapore.
}

The circadian control of glucose metabolism is well recognized, whereby glucose tolerance is significantly lower during the evening as compared to the morning ${ }^{(1,2)}$. Given the increasing prevalence of type 2 diabetes in Asia, considerations must be made not only to the carbohydrate quality but also to the timing of carbohydrate consumption.

This study was designed to assess the effect of low and high glycaemic index (GI) test meals consumed at breakfast or at dinner. All subjects were of Chinese origin, from 50 to 70 years old and had a fasting glycaemia $\leq 7 \mathrm{mmol} / \mathrm{L}$. Postprandial serum glucose concentrations were measured for up to 3 hours. Thirty-four subjects were included in the per-protocol analysis. The prevalence of subjects with glucose values over $7.8 \mathrm{mmol} / \mathrm{L}$ two hours after test meal consumption and onwards (impaired glucose tolerance threshold) were evaluated following the 4 interventions.

High GI test meals, irrespectively if eaten at breakfast or at dinner, led to an increased glycaemic response (GR) compared to low GI test meals (see results in table below). Interestingly, the GR to the low GI test meal eaten for dinner was $55 \%$ greater than the GR to the same meal eaten at breakfast $(\mathrm{p}<0.0001)$. A similar difference was observed when comparing the high GI test meal consumed at dinner versus breakfast $(+59 \%, \mathrm{p}<0.0001)$.

\begin{tabular}{lccc}
\hline $\begin{array}{l}\text { Intervention } \\
\text { Breakfast High GI }\end{array}$ & $\begin{array}{c}\text { Mean iAUC glucose } \\
(\mathrm{mmol} / \mathrm{L} * 180 \mathrm{~min})\end{array}$ & SD & $\begin{array}{c}\text { p-value } \\
\text { High vs Low GI }\end{array}$ \\
Breakfast Low GI & 482.0 & 192.5 & $\begin{array}{c}\text { B-value } \\
\text { Breakfast high vs } \\
\text { low GI: } p=0.0001\end{array}$ \\
Dinner High GI & 378.6 & 158.8 & $\begin{array}{l}\text { Breakfast high GI vs dinner high } \\
\text { GI: } p<0.0001\end{array}$ \\
Dinner Low GI & 766.4 & 320.6 & Dinner high vs \\
low GI: $p=0.0002$ & Breakfast low GI vs dinner low \\
\hline
\end{tabular}

The prevalence of subjects with glucose concentrations over $7.8 \mathrm{mmol} / \mathrm{L} 2$ hours following the test meal and onwards was 2 to 3 times greater when the high or low GI test meal was consumed in the evening compared to the morning $(\mathrm{p}<0.0005)$.

Our results show that the postprandial GR to the same meal is significantly increased in the evening. Therefore, from the perspective of diabetes prevention, the quality and quantity of carbohydrates eaten at dinner should be well controlled.

1. Gibbs M, Harrington D, Starkey S et al. (2014) Clin Nutr 33, 889-94

2. Morgan LM, Aspostolakou F, Wright J et al. (1999) Ann Clin Biochem 36, 447-50 